\title{
The cytoskeleton of chondrocytes of Sepia officinalis (Mollusca, Cephalopoda): an immunocytochemical study
}

\author{
F. Leone, A. Bairati, L. Vitellaro-Zuccarello \\ Dipartimento di Scienze Biomolecolari e Biotecnologie, University of Milan, Italy
}

(C)2004, European Journal of Histochemistry

Our previous electron microscope study showed that chondrocytes from cephalopod cartilage possess a highly developed cytoskeleton and numerous cytoplasmic processes that ramify extensively through the tissue. We have now carried out a light microscope immunocytochemical study of chondrocytes from the orbital cartilage of Sepia officinalis to obtain indications as to the nature of the cytoskeletal components. We found clear positivity to antibodies against mammalian tubulin, vimentin, GFAP, and actin, but not keratin. The simultaneous presence of several cytoskeletal components is consistent with the hypothesis that cephalopod chondrocytes have the characteristics of both chondrocytes and osteocytes of vertebrates, which endow the tissue as a whole with some of the properties of vertebrate bone. We confirm, therefore, the presence in molluscs of the ubiquitous cytoskeletal proteins of metazoan cells that have remained highly conserved throughout phylogenetic evolution.

Key words: cytoskeleton molecules,chondrocytes, Sepia officinalis, immunocytochemistry.

Correspondence: Prof. Aurelio Bairati, Dip. Scienze Biomolecolari e Biotecnologie, via Celoria 26, 20133 Milan, Italy.

Phone: international +39.02 .50314884 .

Fax: international +39.02.50314881.

E-mail: aurelio.bairati@unimi.it

Paper accepted on September 18, 2003

European Journal of Histochemistry

2004; vol. 48 issue 2 [Apr-Jun]: 159-166
A unique feature of the chondrocranial cartilage of cephalopods is the presence of chondrocytes possessing long ramifying cytoplasmic processes. These cells were described by Del Rio-Hortega (1918) and considered to be similar to neurons, glia and osteocytes of vertebrates. Furthermore, the metal staining techniques he used revealed the presence of filaments within the cytoplasm similar to those observed in vertebrate neurons. Rio-Hortega, in fact, concluded that these filaments were analogous to neurofibrils.

We described the ultrastructural morphology of these chondrocytes in a comprehensive study (Bairati et al., 1998) noting the presence of microtubules and filaments. While the microtubules and microfilaments had ultrastructures that enabled us to conclude confidently that they were composed of tubulin and actin respectively, the nature of the intermediate filaments was unclear from their morphology, although by analogy with vertebrate chondrocytes (Benjamin et al., 1994) we presumed that they contain vimentin.

We have now carried out a detailed light-microscope immunocytochemical study of the cytoskeleton of Sepia chondrocytes using antibodies against the typical cytoskeletal constituents of vertebrate cells (anti-tubulin, anti-vimentin, anti-GFAP, antikeratin, and anti-actin). We also used tetramethylrhodamine isothiocyanate (TRITC)-labelled phalloidin to investigate filamentous actin. Our aim was to determine the extent of homology between the cytoskeletal components of Sepia chondrocytes and those in the taxonomically distant vertebrates, hoping to gain insights into the evolution of these macromolecules.

\section{Materials and Methods}

\section{Tissue preparation}

Seven adult specimens of Sepia officinalis (20 $\mathrm{cm}$ ) were processed as soon as possible after catch- 
ing. Small fragments (about $2 \mathrm{~mm}$ thick) dissected from the orbital cartilage were fixed at room temperature in either $4 \%$ paraformaldehyde in $0.1 \mathrm{M}$ phosphate-buffered saline, $\mathrm{pH} 7.4$ (PBS) for $6 \mathrm{~h}$, or $2 \%$ glutaraldehyde plus $0.7 \%$ ammonium hexachlororuthenate (RHT) in $0.1 \mathrm{M}$ phosphate buffer (PB), $\mathrm{pH} 7.2$ for $2 \mathrm{~h}$.

Some blocks were cryoprotected with increasing concentrations ( $10 \%, 15 \%, 20 \%)$ of sucrose solution and with $20 \%$ sucrose $/ 5 \%$ glycerol in $0.1 \mathrm{M}$ $\mathrm{PB}, \mathrm{pH} 7.4$ and embedded in Tissue-Tek OCT Compound (Miles Scientific, IL, USA). These blocks were then frozen by immersion in liquid nitrogen and stored at $-80^{\circ} \mathrm{C}$. Sections were cut at $10 \mu \mathrm{m}$ on a cryostat (Leica) and collected on slides coated with 3-aminopropyltriethoxyethylsilane, airdried and kept frozen pending use. Other cartilage blocks were dehydrated in acetone and embedded in paraffin.

\section{Immunocytochemistry}

Cryostat or paraffin-embedded dewaxed sections were treated sequentially with $0.05 \mathrm{M} \mathrm{N} \mathrm{H}_{4} \mathrm{Cl}$ in $\mathrm{PB}$ for $1 \mathrm{~h}, 0.3 \% \mathrm{H}_{2} \mathrm{O}_{2}$ in methanol for $30 \mathrm{~min}, 10 \%$ bovine serum albumin (BSA) in PBS for $1 h$, and incubated overnight at $4^{\circ} \mathrm{C}$ with primary antibodies. Microwave pretreatment (frequency $2.45 \mathrm{GHz}$, $750 \mathrm{~W}$ ) was applied to improve tubulin immunoreactivity in paraffin sections.

The antibodies used were mouse monoclonal antibodies against: chicken gizzard actin ( $1: 100$, Amersham, UK), pig vimentin (1:50), human glial fibrillary acid protein (1:200, GFAP, Boehringer, Mannheim, 1:200), human pan cytokeratin (1:200); and rabbit polyclonal antibodies against: human vimentin (1:40), human GFAP (1:150), chicken tubulin (1:50). Unless otherwise indicated, antibodies were from Sigma.

Antigen-antibody reactions were visualized using the avidin-biotin-peroxidase complex $(A B C)$ technique (Hsu et al., 1981). This involved sequential incubation in biotinylated secondary antibodies (1:200, Vector Laboratories, Burlingame, CA, USA) for $2 \mathrm{~h}$ at room temperature, followed by avidin-biotin complex (Vectastain ABC kit, Vector Laboratories) for $75 \mathrm{~min}$. The reaction was visualized with diaminobenzidine tetrahydrochloride (DAB, $0.075 \%$ in $0.05 \mathrm{M}$ TRIS- $\mathrm{HCl}$ buffer, $\mathrm{pH}$ 7.3 , with addition of $0.02 \% \mathrm{H}_{2} \mathrm{O}_{2}$ ) as a chromogen.

For immunofluorescent revelation, methanol$\mathrm{H}_{2} \mathrm{O}_{2}$ treatment was omitted, and the antigen-anti- body reaction was revealed using fluorescein isothiocyanate (FITC)-conjugated secondary antibody (1:50, Sigma). Sections were mounted in PBSglycerol 1:3 and examined under a Leica DMR fluorescence microscope equipped with the following combinations of filters: BP 450-490, FT 510, LP 520 for FITC; BP 546,FT 580, LP 590 for TRICT.

\section{Fluorescence microscopy of F-actin}

Cryostat sections of cartilage segments fixed in 4\% paraformaldehyde were stained with TRITClabelled phalloidin (5 U/mL in PBS, Sigma) for 10 min at $4^{\circ} \mathrm{C}$. Excess TRITC-phalloidin was removed by several washes with PBS and sections were mounted in PBS-glycerol.

\section{Controls}

In control sections, incubation with primary antibody was omitted. The specificity of the antibodies was tested on appropriate vertebrate tissue: antiactin on chicken skeletal muscle; anti-pan cytokeratin and anti-vimentin on human skin; and antitubulin and anti-GFAP on rat cerebellar cortex.

\section{Results}

Chondrocyte staining was highly variable and sometimes extremely weak, so various preparation methods were tried in order to demonstrate the location of a given antigen.

\section{TRITC-phalloidin and anti-actin}

After treatment with TRITC-phalloidin (Figure 1 ), both globular chondrocytes of the inner zone, and finely elongated cells of the outer zone of the orbital cartilage showed fluorescence located mainly at the periphery of the cell body and the origins of the cytoplasmic processes (Figure $1 \mathrm{~A}$ ). Furthermore, numerous small fluorescent spots were observed along cell surfaces (Figure 1A, 1B). Staining with monoclonal antibody against chicken actin produced more intense and extensive staining of chondrocyte cell bodies, and was also present to variable extents in the cytoplasmic processes (Figure 1C).

\section{Anti-vimentin antibodies}

The staining reaction of chondrocytes to antivimentin antibodies varied in intensity with the type of antibody (anti-pig or anti-human vimentin) and the sample preparation method (Figure 2A-D). In 

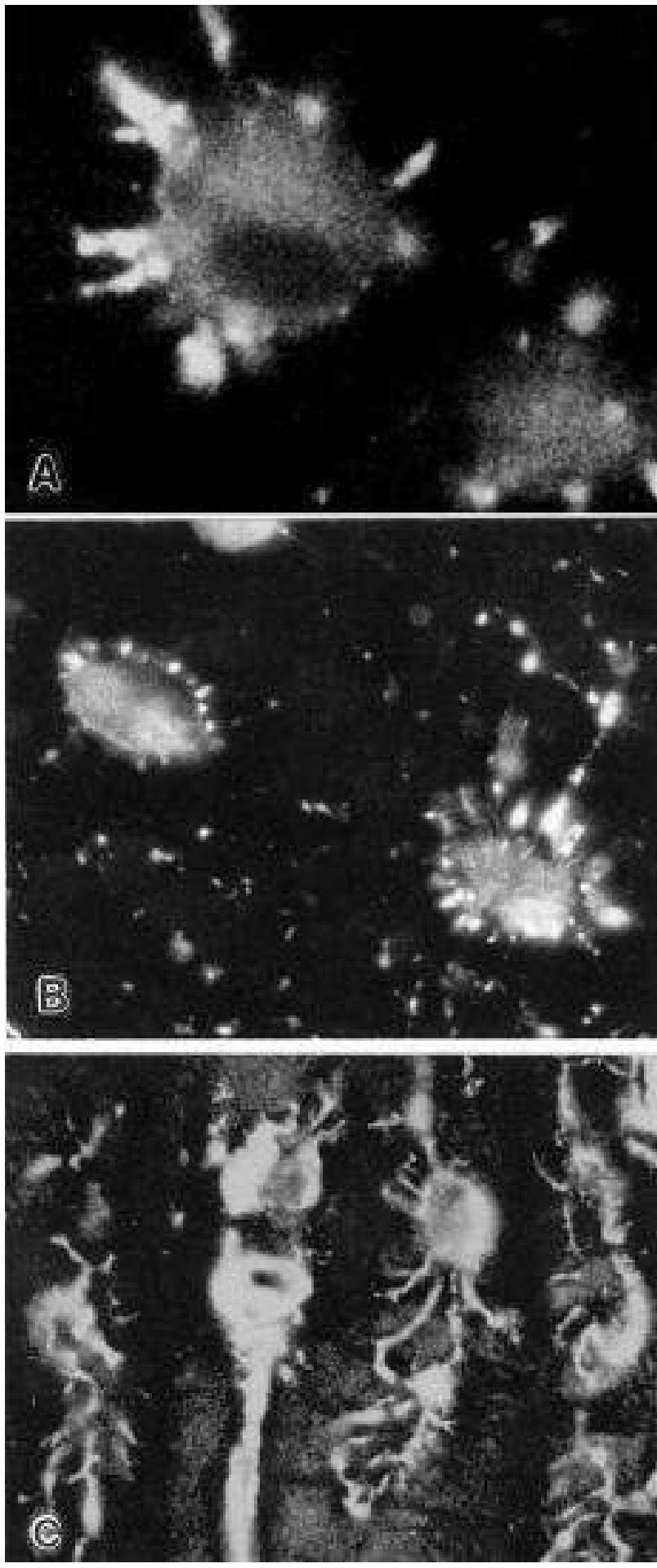

Figure 1. Cryosections of Sepia orbital cartilage fixed in paraformaldehyde. A (x 300), B (x 200): staining with TRITCphalloidin viewed under fluorescent microscope. C: indirect immunostaining with antibody against chicken actin (x 200).

paraformaldehyde-fixed cryostat sections treated with monoclonal antibodies against pig vimentin, the initial tracts of the largest cytoplasmic process, as well as the cell bodies, were intensely stained; within the cell bodies areas of reticular staining were visible (Figure $2 \mathrm{~A}$ ). Use of polyclonal antihuman vimentin on glutaraldehyde-fixed cryosections produced weak background staining in chondrocytes against which short tracts around the nucleus and at the cell periphery stood out as more intensely stained (Figure 2B). The flattened chondrocytes of the outer zone of the cartilage were more uniformly and intensely stained (Figure 2C). Use of polyclonal anti-human vimentin on glutaraldehyde-fixed, paraffin-embedded sections produced intense staining both in cell bodies and cytoplasmic processes; staining was particularly intense in samples fixed with glutaraldehyde/RHT (Figure 2D).

\section{Anti-GFAP antibodies}

Monoclonal antibody against human GFAP on paraformaldehyde-fixed cryostat sections did not produce any reaction with DAB. Polyclonal antiGFAP antibody produced a weak granular (dusty) positivity in chondrocyte cell bodies.

On glutaraldehyde fixed cryosections, anti-GFAP polyclonal antibodies intensely stained elongated tracts at the cell body periphery (Figure 3A), but did not stain cytoplasmic processes.

Much more intense and extensive staining was obtained in cell bodies and cytoplasmic processes on glutaraldehyde/RHT-fixed cryosections, particularly after intensification with the $A B C$ method (Figure 3B). On paraffin-embedded samples fixed with glutaraldehyde/RHT, staining was even more intense, with areas of reticulated positivity both in cell bodies and cytoplasmic processes (Figure $3 \mathrm{C}$ ).

\section{Anti-pan cytokeratin antibodies}

Anti-pan cytokeratin antibodies - which reacted with mammalian epidermis - did not produce any reaction on any Sepia sample.

\section{Anti-tubulin antibodies}

Polyclonal antibodies against chicken tubulin produced variable staining reactions in relation to the sample preparation method.

On glutaraldehyde/RHT fixed cryostat sections treated with polyclonal antibody, staining picked out elongated tracts and dusty or granular areas at the cell body periphery; cytoplasmic processes were weakly stained (Figure 4A). Staining was more intense and extensive in paraffin-embedded sections, in both cell bodies and cytoplasmic processes 


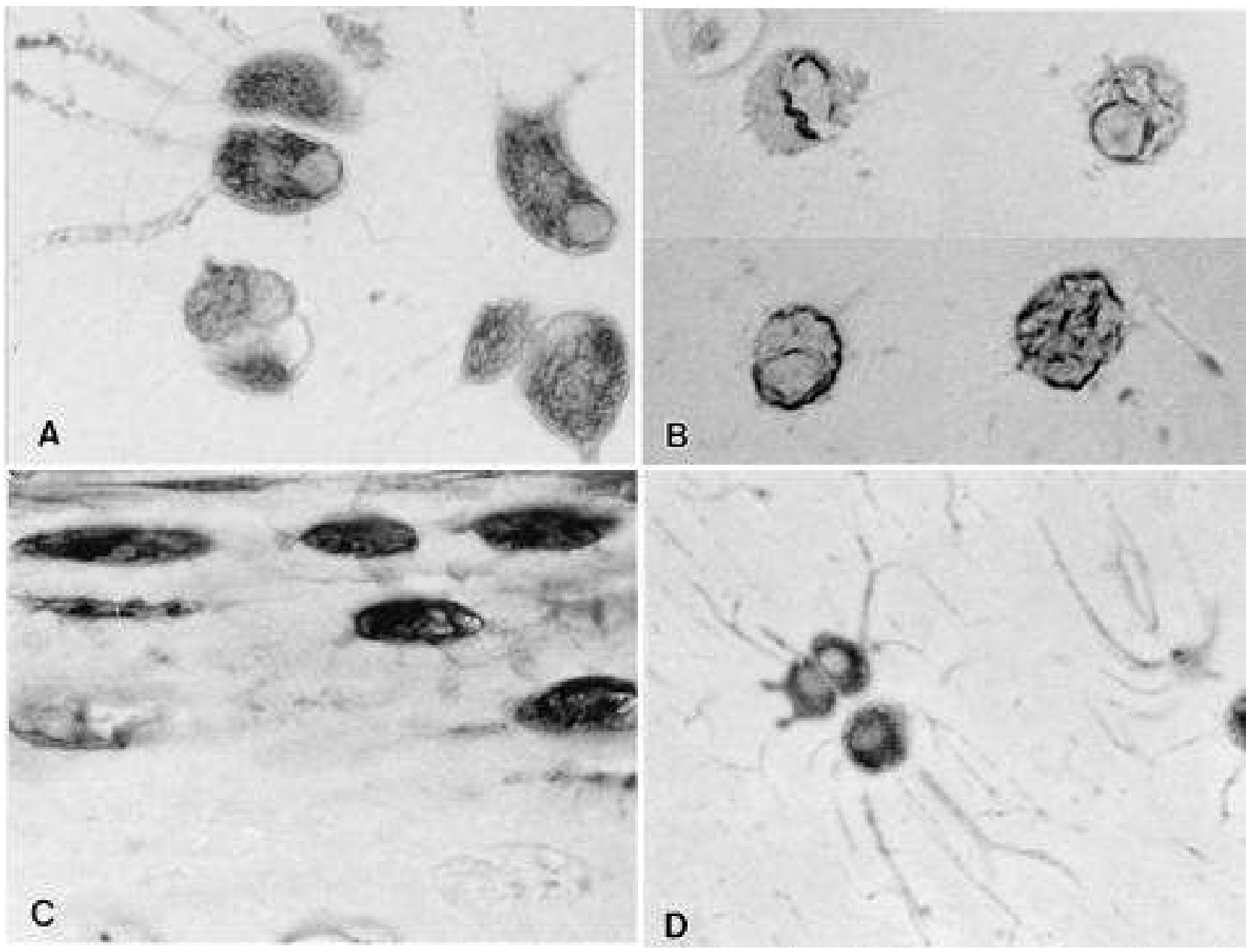

Figure 2. Sections of Sepla orbital cartilage treated with anti-vimentin antibodies. A: paraformaldehyde-fixed cryosections reacted with monoclonal antibody against pig vimentin (x 750). B: glutaraldehyde-fixed cryosections reacted with polyclonal antibody against human vimentin (x 750). C: chondrocytes from outer zone cartilage treated as in B (x 630). D: paraffin-embedded section fixed in glutaraldehyde RHT and treated with polyclonal antibody against human vimentin (x 550).

(Figure 4B), particularly after microwave treatment (Figure 4C).

\section{Controls}

No positivity was ever observed, either with DAB or fluorescent staining, on any of the control sections not treated with primary antibody. All antibodies were checked for specificity by reaction with the specific tissues containing the antigens used to raise the antibodies. In all cases, the expected specific cellular positivity was observed.

\section{Discussion}

\section{General aspects}

Our use of a variety of fixation and preparation methods allowed us to observe two main morphological patterns of immunoreactivity: a) tracts and filaments, the latter sometimes forming a network, and (b) extensive areas of uniform finely granular (dusty) staining. The morphology of the filamentous immunoreactivity corresponded closely to the filamentous entities observed in the light microscope by silver salt staining (Del Rio-Hortega, 1918), and which, in the electron microscope, are revealed as intermediate filaments, microtubules, and microfilaments (Bairati et al., 1998). Aldehydes, particularly glutaraldehyde with $\mathrm{RHT}$, proved the best fixatives in that they allowed good immunostaining and at the same time conserved cell body morphology and cytoplasmic processes without shrinkage and detachment of the cells from the extracellular matrix - as occur with traditional histological methods (Del RioHortega, 1918). Use of RHT is reported to have similar stabilising effects on GAGs and PGs of the pericellular extracellular matrix (Hunziker et al., 

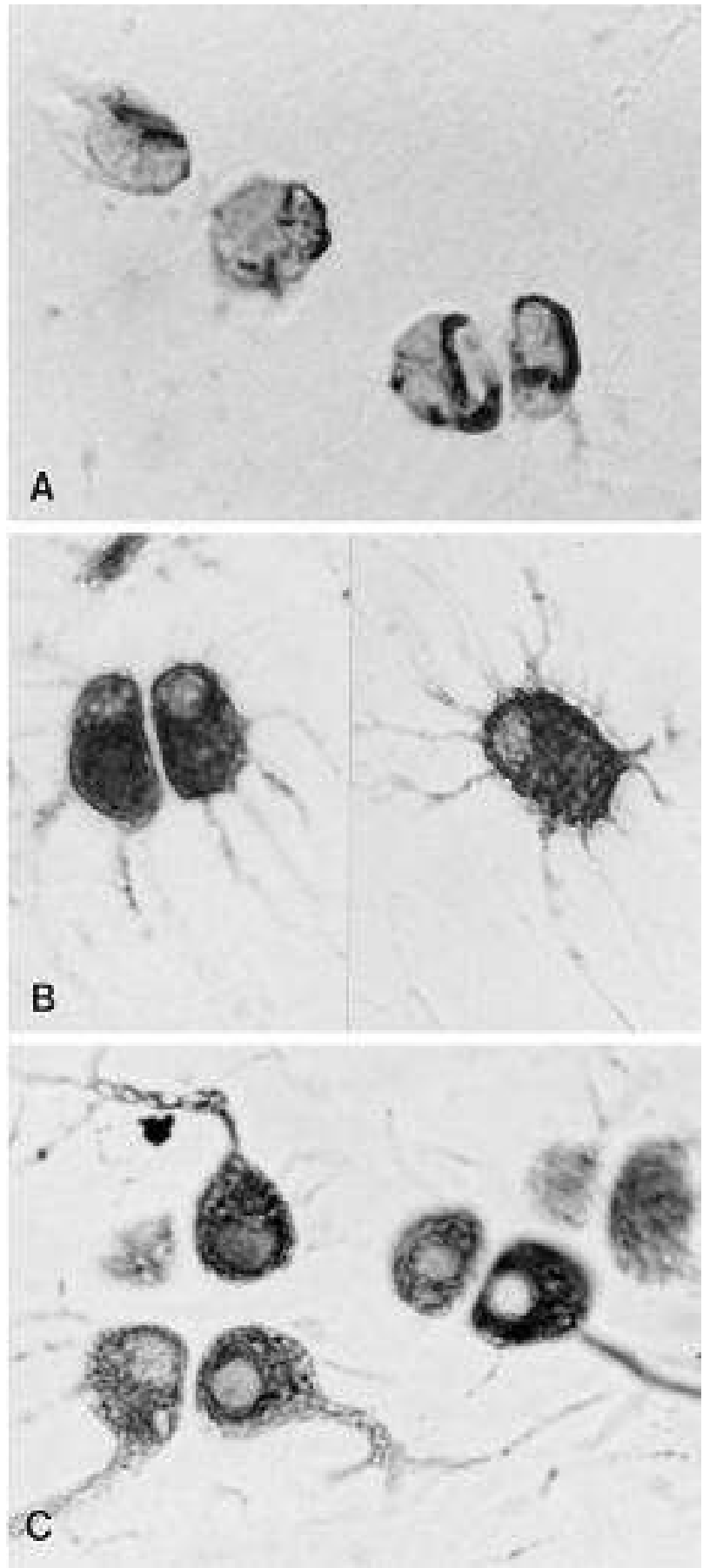

Figure 3. Sections of Sepla orbital cartilage treated with antiGFAP antibodies. A: glutaraldehyde-fixed cryosections and polyclonal antibody against human GFAP (x750). B: glutaraldehyde/RHT-fixed cryosection reacted with polyclonal antibody against human GFAP $(x 750)$. C: paraffin-embedded section fixed and stained as in B (x750).

1982). Glutaraldehyde is known to stabilize microtubules by inhibiting the depolymerisation that occurs during freezing (Osborne and Weber, 1982).

The technique of paraffin embedding produced more intense and diffuse staining than cryosection-

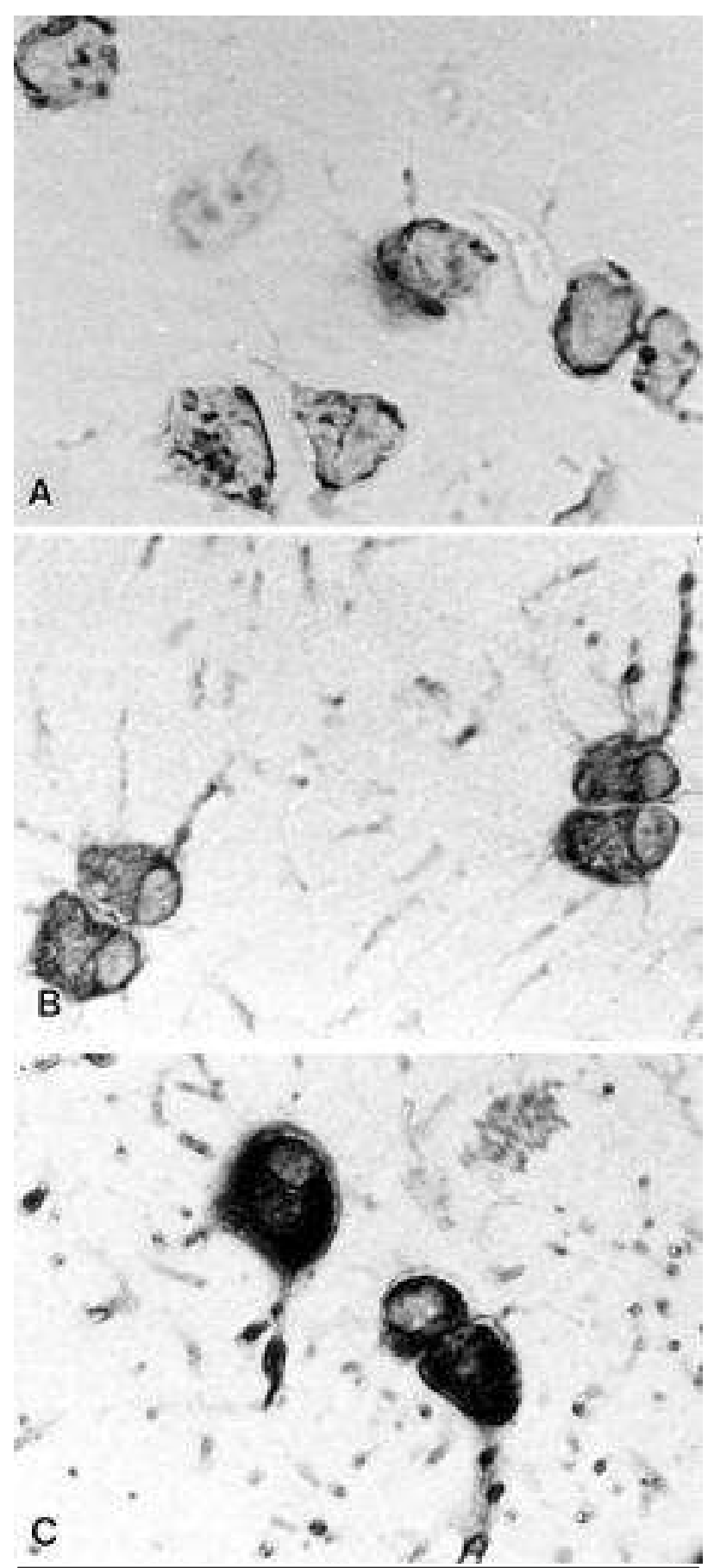

Figure 4.Sections of Sepla orbital cartilage treated with polyclonal anti-tubulin antibodies. A: glutaraldehyde/RHT-fixed cryosection (x 750). B: paraffin embedded section fixed as in $A(x 750)$. C: microwave-treated paraffin section of sample fixed as in $A(x$ 750). *

ing; however, the cells typically showed coarse structures indicating artefactual aggregation of cytoplasmic components. Like Shi et al. (1991), we found that prior microwave exposure intensified immune reactivity. 


\section{Actin microfilaments}

Areas positive to TRITC-phalloidin were often present at the periphery of the cell body and at the origin of the cytoplasmic processes; such areas correspond to those in electron microscope preparations (Bairati et al., 1998) where irregular aggregations of microfilaments are present and where various cytoskeletal structures converge. We propose that the small fluorescent spots observed along the surface of the cells represent sites of focal adhesion between the cell and the extracellular matrix (Bairati et al. 1998, Fig. 5). At such sites, actin microfilaments interact with various actin-related proteins (Burridge et al.,1988) to form the cytoplasmic dense bodies visible also in the light microscope.

We found positivity to antibodies against chicken actin within cytoplasmic processes, indicating the extensive presence of microfilaments within these processes. In this respect, Sepia chondrocytes are similar to vertebrate osteocytes: actin microfilaments have been demonstrated in osteocyte cytoplasmic processes using the confocal microscope with phalloidin (Jones and Taylor, 1990) and by immunostaining (Tanaka-Kamioka et al., 1998). Actins, widespread in invertebrate cells (Goodson and Hawse, 2002), and now demonstrated immunochemically in cephalopods, constitute one of the oldest protein families of eukaryotes and one of the most highly conserved during phylogenetic evolution.

\section{Intermediate filaments: vimentin and GFAP}

Our data clearly show that vimentin- and GFAPlike proteins are expressed in Sepia chondrocytes. We propose that the uniform positivity within cell bodies represents pools of these molecules in the cytosol; while the tracts, filaments and reticular structures indicate the presence of filaments in various stages of assembly. The filaments and reticular structures picked out by these antibodies correspond well to the forms observed by Del RioHortega (1918) in the light microscope, using silver staining, and those we have described in the electron microscope of the same cells (Bairati et al., 1998). The larger tracts of immunopositivity observed in the present study seem to correspond to the larger and denser bundles we observed in the electron microscope. Our electron microscope study also showed that, within the cytoplasmic processes, intermediate filaments were present as individual elements interspersed between bundles of microtubules: and these correspond well to the immunopositivity for vimentin and GFAP in cytoplasmic processes most convincingly revealed in paraffin-embedded sections using intensification.

This study therefore provides additional evidence that the highly conserved proteins that form intermediate filaments are present in cephalopods, as previously shown by the electron microscope and immunocytochemical studies of Bartnik and Weber (1989) and the molecular cloning study of Erber et al. (1998). In vertebrates, vimentin is considered to be expressed in mesenchyme-derived cells, while GFAP is expressed almost exclusively in glia. Nevertheless, GFAP has also been described in mesenchyme cells (Kasantikul and Shuangshoti, 1989; Kasper and Stosiek, 1990), mainly in certain disease conditions (Viale and al., 1988), but also in cartilage cells (Benjamin et al., 1994) where it is expressed together with vimentin and cytokeratin.

It is possible, however, that both vimentin and GFAP filaments are not present in these cells as individual elements, but only a single type of molecule exists whose conserved epitopes are recognized by both vimentin and GFAP polyclonal antibodies. In fact, Weber et al. (1991) have proposed that these proteins are derived from a single ancestral protein closely similar to nuclear lamins. In our opinion, it is likely that a molecule closely homologous to vertebrate GFAP is present in molluscs, although this requires confirmation by sequencing. It would be interesting from the evolutionary point of view to determine whether heterotypic intermediate filaments are present in molluscs, just as they are in vertebrates (Herrmann and Aebi, 1999).

\section{Microtubules and tubulin}

The immunocytochemical findings of the present study provide further evidence for a close homology between cephalopod and vertebrate tubulins. Like actin, tubulins are ubiquitous components of eukaryotic cells whose structure has been highly conserved during evolution. However, cartilage cells do not usually contain the abundance of microtubules observed in Sepia chondrocytes, and it seems evident that this abundance is related to the ability of these cells to form extensive ramifications of cytoplasmic processes. In fact, we suggest that tubulin expression may be the principal differentiation characteristic of cephalopod chondrocytes which enables them to perform their characteristic 
structural, dynamic and metabolic functions in cartilage (Bairati et al., 1998).

\section{Comparative aspects}

Our previous light microscope (Bairati et al., 1987) and ultrastructural studies (Bairati et al., 1998) indicated that cephalopod chondrocytes possess structural characteristics typical of both the chondrocytes and osteocytes of vertebrates. The chondrocytes of vertebrates express vimentin, cytokeratin and GFAP as main cytoskeletal components (Benjamin et al., 1994), while actin and tubulin are also present in the cytosol. Vertebrate osteocytes not only express vimentin (Shapiro et al., 1995) but also actin concentrated in the cytoplasmic processes (Jones and Taylor, 1990; Aarden et al., 1994). The present immunocytochemical study provides evidence that tubulin, vimentin, GFAP and actin are present in cephalopod chondrocytes; however, microtubules were perticularly abundant in relation to the extensive development of cytoplasmic processes that caharacterise these cells and permit them to form a cartilagenous tissue in many respects similar to the bone of vertebrates (Bairati et al., 1987) although it lacks mineralisation.

\section{Acknowledgments}

Financial support was provided by funds from FIRST. We would like to thank Don Ward for the help with the English. We are grateful to R. De Sanctis and the staff of the Stazione Zoologica "A.Dornh" of Naple for supplying the animals.

\section{References}

Aarden EM, Burger EH, Nijweide PJ. Function of osteocytes in bone. $\mathrm{J}$ Cell Biochem 1994;55:287-99.

Bairati A, De Biasi S, Cheli F, Oggioni A. The head cartilage of cephalopods. I. Architecture and ultrastructure of the extracellular matrix. Tissue Cell 1987;19:673-85.
Bairati A, Comazzi M, Gioria M, Rigo C. The ultrastructure of chondrocytes in the cartilage of Sepia officinalis and Octopus vulgaris (Mollusca, Cephalopoda). Tissue Cell 1998;30:340-51.

Bartnik E, Weber K. Widespread occurence of intermediate filaments in invertebrates; common principles and aspects of diversion. Eur $\mathrm{J}$ Cell Biol 1989; 1:17-33.

Benjamin M, Archer C, Ralphs JR. Cytoskeleton of cartilage cells. Microsc Res Tech 1994;28:372-7.

Burridge K, Fath K, Kelly T, Nuckolls G, Turner C. Focal adhesions: trasmembrane junctions between the extracellular matrix and the cytoskeleton. Ann Rev Cell Biol 1988;4:487-525.

Del Rio-Hortega P. Sobre la fine textura del cartilago de los cefalòpodos. Tra Lab Inv Biol Univ Madrid 1918;16:185-212.

Erber A, Riemer D, Bovenschulte M, Weber K. Molecolar phylogeny of metazoan intermediate filament proteins. J Mol Evol 1998;47:75162.

Goodson HV, Hawse WF. Molecular evolution of the actin family. J Cell Sci 2002;115:2619-22.

Herrmann $\mathrm{H}$, Aebi U. Intermediate filament assembly: temperature sensitivity and polymorphism. Cell Mol Life Sci 1999;55:1416-31.

Hunziker EB, Herrmann W, Schenk RK. Improved cartilage fixation by ruthenium hexammine trichloride (RHT).J Ultrastr Res 1982;81:112.

Hsu SM, Raine L, Fanger $\mathrm{H}$. Use of avidin-biotin-peroxidase complex $(A B C)$ in immunoperoxidase techniques: a comparison between $A B C$ and unabeled antibody (PAP) procedures. J Histochem Cytochem 1981;29:557-80.

Kasantikul V, Shuangshoti S. Positivity to glial fibrillar acidic protein in bone, cartilage and chordoma. J Surg Oncol 1989;41:22-6.

Kasper M, Stosiek P. Detection of GFAP in vertebral fibrocartilage in human fetal and newborn tissue. Acta Histochem 1990;88:25-7.

Jones SJ, Taylor ML. Confocal fluorescence microscopy: some applications in bone cell biology. J Microsc 1990;158:249-59.

Osborne M, Weber K. Immunofluorescence and immunocytochemical procedures with affinity purified antibodies: tubulin-containing structures. Meth Cell Biol 1982;24:97-132.

Shapiro F, Cahill C, Malatantis G, Nayak RC. Transmission electron microscopic demonstration of vimentin in rat osteoblast and osteocyte cell bodies and processes using the immuno-gold technique. Anat Rec 1995;241:39-48.

Shi $\mathrm{S}$, Key ME, Kalra KL. Antigen retrieval in formalin-fixed, paraffin embedded tissue: an enhancement method for immunohistochemical staining based on microwave oven heating of tissue sections. J Histochem Cytochem 1991;39:741-8.

Tanaka K, Kamioka H, Ris H, Lim SS. Osteocyte shape is dependent on actin filaments and osteocyte processes are unique actin-rich projections. J Bone Miner Res 1998;13:1555-68.

Viale G, Doglioni C, Dell'Orto P, Zanetti G, Iuzzolino P, Bontempini, L, Coggi G. Glial fibrillary acidic protein immunoreactivity in human respiratory tract cartilages and pulmonary chondromatous hamartomas. Am J Pathol 1988;133:363-73.

Weber K, Riemer D, Dodemont H. Aspects of the evolution of the lamin/intermediate filament protein family: a current analysis of invertebrate filament proteins. Biochem Soc Trans 1991;19:1021-3. 
F. Leone et al. 